OPEN ACCESS

Edited by:

Manisha Yadav,

University of Delhi, India

Reviewed by:

Avishek Mitra,

Oklahoma State University,

United States

Jianjun Sun,

The University of Texas at El Paso,

United States

*Correspondence:

Xuelian Zhang

xuelianzhang@fudan.edu.cn

Specialty section:

This article was submitted to Molecular Bacterial Pathogenesis,

a section of the journal

Frontiers in Cellular

and Infection Microbiology

Received: 07 September 2020

Accepted: 31 October 2020

Published: 26 November 2020

Citation:

Qian J, Chen R, Wang H and Zhang X (2020) Role of the PE/PPE Family in

Host-Pathogen Interactions and

Prospects for Anti-Tuberculosis Vaccine and Diagnostic Tool Design.

Front. Cell. Infect. Microbiol. 10:594288.

doi: 10.3389/fcimb.2020.594288

\section{Role of the PE/PPE Family in Host- Pathogen Interactions and Prospects for Anti-Tuberculosis Vaccine and Diagnostic Tool Design}

\author{
Jianing Qian ${ }^{1}$, Run Chen ${ }^{1}$, Honghai Wang ${ }^{1}$ and Xuelian Zhang ${ }^{1,2 *}$ \\ ${ }^{1}$ State Key Laboratory of Genetic Engineering, School of Life Science, Fudan University, Shanghai, China, ${ }^{2}$ Shanghai \\ Engineering Research Center of Industrial Microorganisms, Fudan University, Shanghai, China
}

The pe/ppe genes are found in pathogenic, slow-growing Mycobacterium tuberculosis and other M. tuberculosis complex (MTBC) species. These genes are considered key factors in host-pathogen interactions. Although the function of most PE/PPE family proteins remains unclear, accumulating evidence suggests that this family is involved in M. tuberculosis infection. Here, we review the role of PE/PPE proteins, which are believed to be linked to the ESX system function. Further, we highlight the reported functions of PE/ PPE proteins, including their roles in host cell interaction, immune response regulation, and cell fate determination during complex host-pathogen processes. Finally, we propose future directions for PE/PPE protein research and consider how the current knowledge might be applied to design more specific diagnostics and effective vaccines for global tuberculosis control.

Keywords: PE/PPE proteins, Mycobacterium tuberculosis, macrophages, host-pathogen interaction, vaccine

\section{INTRODUCTION}

Mycobacterium tuberculosis (Mtb), the causative pathogen of tuberculosis (TB), is an extremely successful intracellular pathogen. The interactions between Mtb and host immune system determine the outcome of Mtb infection. PE/PPE families are seemingly related to mycobacteria pathogenicity, as its members are abundant in pathogenic mycobacteria (Akhter et al., 2012; Li et al., 2019) and less present in nonpathogenic mycobacteria (McGuire et al., 2012). Previous reviews have discussed the pe/ppe genes evolution (Fishbein et al., 2015), the expression and regulatory role of PE/ PPE proteins (Li et al., 2019), as well as the relation with virulence and host cell fate (Yu et al., 2019).

Given the importance of the PE/PPE family in host-pathogen interactions, herein, we summarize the latest experimental advances in PE/PPE protein interactions with host cells and provide a comprehensive overview of the involvement in macrophage processing of Mtb, such as adhesion, receptor interactions, immune response, environmental stress resistance, phagocytosis, intracellular survival, and cell fate regulation. This information may contribute to tuberculosis future intervention strategies, such as improved diagnostic tools and vaccine candidates. 


\section{FUNCTIONAL RELATIONSHIPS BETWEEN PE/PPE PROTEINS AND THE ESX SECRETION SYSTEM}

Currently, the original evolution of the pe/ppe gene families remains unclear. However, the ancestral pe/ppe gene family is reportedly related to the ESX (early secretory antigen target 6 system) protein family (Gey van Pittius et al., 2006). The pe/ppe genes seem to have evolved and duplicated in association with the duplication of the five esx gene cluster regions in the Mtb genome (Gey van Pittius et al., 2006; Akhter et al., 2012; Fishbein et al., 2015) that can be inferred from the most primitive ESX-4, which has no PE/PPE proteins among its components, in contrast to the more recent ESX-5, which has two PE (PE18 and PE19) and three PPE (PPE25, PPE26, and PPE27) proteins (Majlessi et al., 2015). Besides, the recently evolved PE_PGRS (polymorphic GC-rich sequences) and PPE_MPTR (major polymorphic tandem repeats) subfamilies are believed to have originated from pe/ppe genes within the ESX-5 cluster (Gey van Pittius et al., 2006).

Recent studies have indicated that the ESX system contributes to $\mathrm{PE} / \mathrm{PPE}$ protein export, and, likewise, ESX system protein secretion is related to that of PE/PPE proteins. Genes in ESX-1 locus that encode secreted proteins EsxA and EsxB are flanked directly upstream by pe35 and ppe68 (Majlessi et al., 2015). PPE68 and PE35 are required for Mtb virulence (Sassetti and Rubin, 2003; Jiang et al., 2016), and PE35 is required for EsxA and EsxB secretion (Brodin et al., 2006; Chen et al., 2013).

ESX -5 is related to the virulence of pathogenic mycobacteria. Deletion of ppe25, pe18, ppe26, ppe27, and pe19 significantly attenuated the virulence in mouse models. Further, ESX-5 inactivation Mtb and Mycobacterium marinum mutants fail to secrete several PE/PPE proteins, many of which are not encoded by the esx -5 locus, suggestive of a loss of the ability to transport PE/ PPE proteins across mycobacterial cell envelope (Bottai et al., 2012). In addition, the expression of PE19 enhances envelope permeability inducing higher pathogenic sensitivity (Ramakrishnan et al., 2016). These results strongly suggest that PE/PPE proteins of ESX-5 locus are required for ESX-5 mediated protein export.

Although, to a lesser extent than ESX-1 and ESX-5, the correlation between ESX-3 and PPE-related functions has also been studied. Products of the esx -3 gene locus, which contains pe5 and ppe4, carry out the essential function of iron/zinc acquisition (Serafini et al., 2009; Siegrist et al., 2009). PE5 forms a heterodimer with PE4 to utilize iron from the intracellular host space (Tufariello et al., 2016). In contrast to other members, the exact function of esx-2, including pe36 and ppe69, remains undefined.

Overall, due to the difficulty of recovering stable soluble recombinant $\mathrm{PE} / \mathrm{PPE}$ proteins, knowledge of their biophysical structure remains insufficient to clarify the secretory interaction between PE/PPE proteins and the ESX system. However, it is worth mentioning that the ESX secretion-associated protein G (EspG), the homolog of the ESX system, recognizes its cognate $\mathrm{PE} / \mathrm{PPE}$ protein, maintaining it in a stable conformation and promoting secretion (Daleke et al., 2012). The crystal structure of the PE25-PPE41-EspG 5 complex yielded valuable information regarding the cross-talk between EspGs and different PE/PPE proteins (Ekiert and Cox, 2014; Korotkova et al., 2014).

\section{ROLE OF PE/PPE PROTEINS IN HOST- PATHOGEN INTERACTIONS}

Based on the different stages of interaction with the host, we summarize the function and localization of PE/PPE proteins in Supplementary Table 1 and highlight the intriguing roles in Figure 1A: (i) mediating immune responses through cell surface adhesion or receptor binding; (ii) surviving under intracellular stress, phagocytosis, and phagolysosome maturation; (iii) determination of cell fate.

\section{Roles of PPE Proteins in Interactions With Host Cells and Immune Regulation}

Surface exposure or secretion into the extracellular environment allows PE/PPE proteins to interact with their host targets directly. Some proteins reportedly interact with receptors like TLR2/4 on the surface of macrophages, activating downstream signaling pathways. The PE9-PE10 complex (Tiwari et al., 2015), PPE39 (Choi et al., 2019), and PE_PGRS5 (Grover et al., 2018) interact with TLR4 to activate downstream signaling and modulate cytokine production. Furthermore, various PE/PPE proteins can interact with TLR2, including PPE26 (Su et al., 2015), PPE32 (Deng et al., 2014), PPE57 (Xu et al., 2015), PPE65 (Qureshi et al., 2019), PE_PGRS33 (Basu et al., 2007; Zumbo et al., 2013; Palucci et al., 2016), and PE_PGRS11 (Bansal et al., 2010). PPE18 can stimulate IL-10 secretion, which might induce Th2 type response via interacting with TLR2 (Nair et al., 2009), and further was defined to inhibit the production of NF- $\mathrm{kB} / \mathrm{rel}-$ mediated pro-inflammatory cytokine by upregulating suppressor of cytokine signaling 3 protein (SOCS3) (Nair et al., 2011). Besides, PE_PGRS17 was found to mature DCs via TLR2 (Bansal et al., 2010) and cause host cell death and cytokine secretion via Erk kinase, eventually enhancing intracellular survival (Chen et al., 2013).

Generally, the binding of PE/PPE proteins to cell surface receptors activates downstream signaling pathways, including NF- $\mathrm{KB}$ and MAPK (p38, JNK, and ERK), which affect cytokine production, leading to a pro-inflammatory or anti-inflammatory response. PPE27 overexpressed strain showed a strengthened ability to induce nitric oxide (NO) and inhibiting IL-6 production, which was abolished by NF- $\mathrm{KB}, \mathrm{p} 38$, and ERK inhibitors (Yang G et al., 2017). PPE39, a PE/PPE protein defined in hypervirulent strain Beijing/K, exhibited its ability to mature DCs and activate Th1 immune response through NF$\kappa \mathrm{B}$ and MAPK, which functioned as TLR4 agonist (Choi et al., 2019). A series of proteins, including PE13 (Li et al., 2016), PE27 (Kim et al., 2016), PPE26 (Su et al., 2015), PPE32 (Deng et al., 2014), PPE44 (Yu et al., 2017), PPE57 (Xu et al., 2015), PE_PGRS11, and PE_PGRS17 (Bansal et al., 2010) similarly regulate the cytokine profile via NF- $\mathrm{KB}$ and MAPK signaling.

$\mathrm{PE} / \mathrm{PPE}$ protein effects on mycobacterial invasion and macrophage phagocytosis have also been suggested. PPE38- 


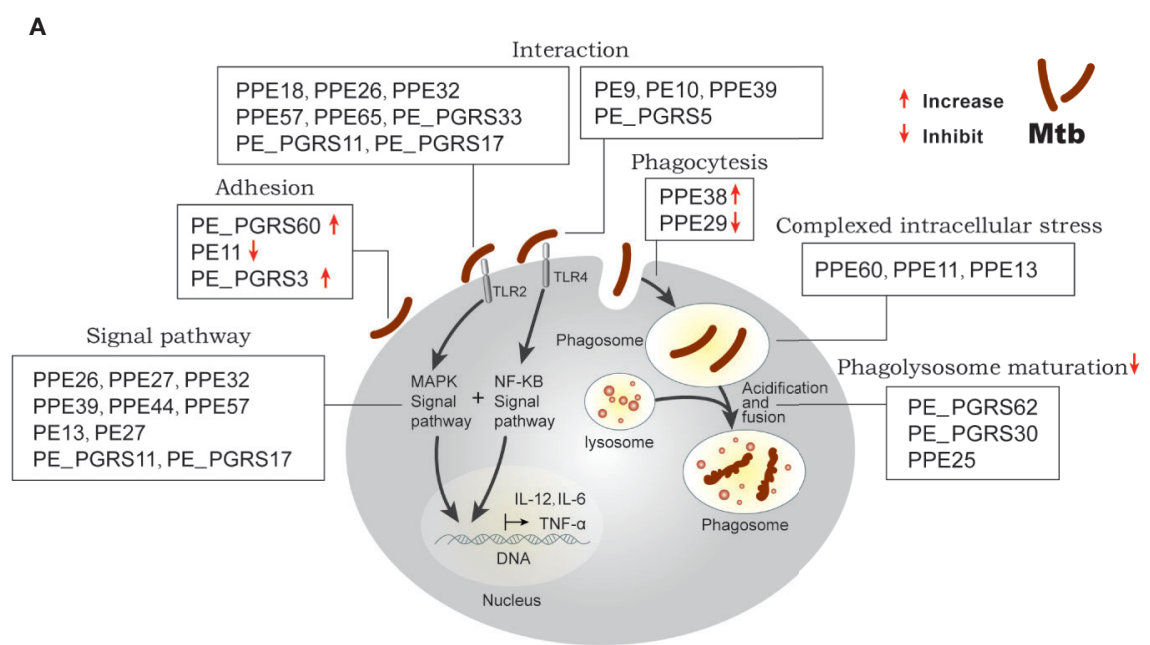

Macrophage

B

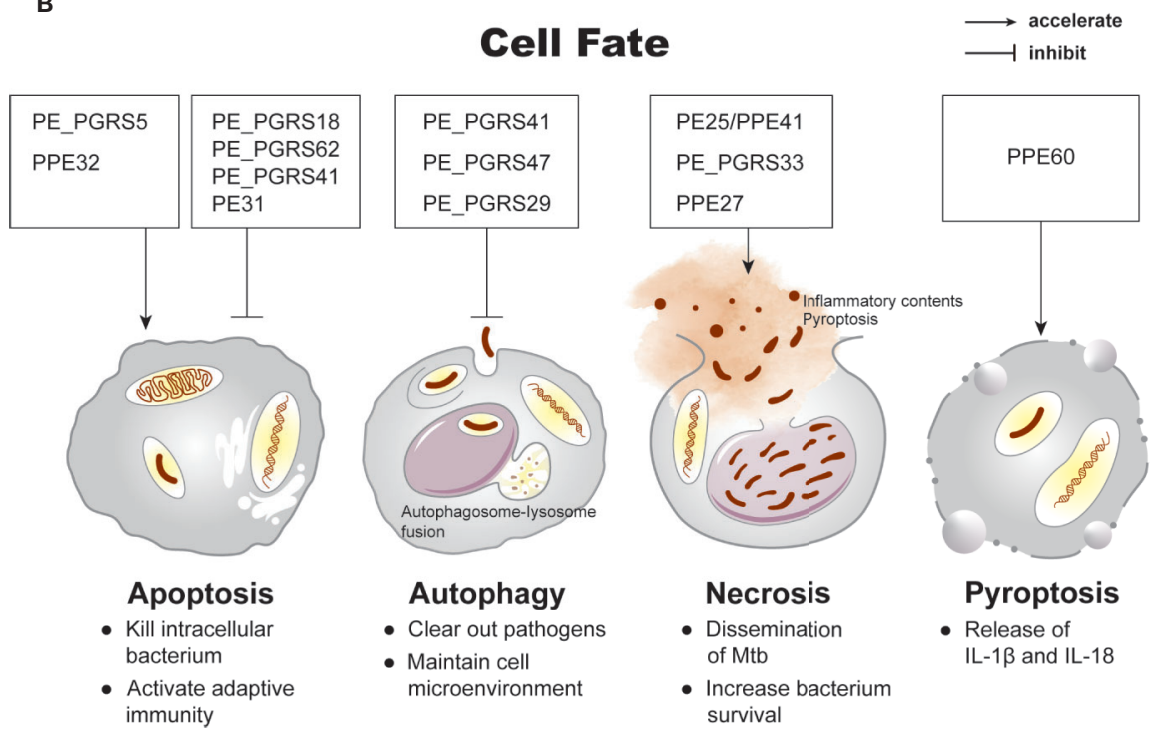

FIGURE 1 | Effects of PE/PPE proteins on the interaction between Mtb and macrophage. (A) PE/PPE proteins in each frame are shown to affect each stage of host-pathogen interaction, including in cell adhesion and binding to TLR2 or TLR4 receptors, mediating downstream immune signal pathway, inhibiting or increasing phagocytosis of the bacilli, surviving under intracellular stress, and inhibiting phagolysosome maturation in macrophage. (B) PE/PPE proteins regulate four major outcomes observed in macrophage after Mtb infection.

mutant of Mycobacterium marinum exhibited significantly higher invasion efficiency (Dong et al., 2012), while the phagocytosis ratio of PPE29 mutants was expectedly reduced (Meng et al., 2017).

Adherence to the cell surface is another prerequisite for bacterial invasion. Recent reports revealed that PE11 knockdown strains could significantly enhance fibronectin attachment protein production, contributing to the attachment to the host extracellular matrix (Rastogi et al., 2017). PE_PGRS60 can bind to fibronectin, which results in enhanced adhesion and invasion (Meena and Meena, 2016).

\section{Roles of PE/PPE Proteins in Intracellular Survival}

Upon entry into macrophages, pathogens adapt to the intracellular environment, such as low $\mathrm{pH}$, reactive oxygen, and nitrogen species, thus creating its own niche. Besides, PPE60 (Gong et al., 2019) and PE13 (Li et al., 2016) can enhance cell resistance to low $\mathrm{pH}$, surface stresses, and antibiotic exposure to increase intracellular survival. PPE11 has also increased early bacterial survival rate under conditions similar to the intracellular macrophage environment, such as 
the presence of lysozymes, acidic, and active nitrogen intermediates (RNI), and maintains a high bacterial load in mouse tissue, worsening organ pathology (Peng et al., 2018).

Once adapted to the harsh conditions, Mtb survives in macrophages by preventing phagosomal acidification and phagosome-lysosome fusion. PE_PGRS30 and PE_PGRS47 knockout strains lost the ability to inhibit phagosome fusion (Iantomasi et al., 2012; Saini et al., 2016). Similarly, overexpressed PE_PGRS62 significantly inhibits phagosome maturation (Huang et al., 2012; Thi et al., 2013; Long et al., 2019). During phagocytosis, the transcription level of PPE25 is upregulated, and PPE25 mutant strain loses its ability to replicate within macrophages and prevent phagosome-lysosome fusion (Jha et al., 2010).

\section{PE/PPE Proteins Are Involved in the Determination of Cell Fate}

Further, PE/PPE proteins are believed to have roles in host defense mechanism which limit Mtb survival or are closely associated with the intracellular persistence and proliferation, eventually inducing host cells three major outcomes as shown in Figure 1B: (i) apoptosis, a form of programmed cell death that is proactively regulated by host cells (Fink and Cookson, 2005); (ii) autophagy, a host degradation system that can resolve infection (Mariño et al., 2014); and (iii) necrosis, a form of passive cell death triggered by external stimuli (Fink and Cookson, 2005).

Cell apoptosis can affect intracellular bacterial viability (Duan et al., 2002). Recent studies indicate that PPE32 (Deng et al., 2016) and PE_PGRS5 (Grover et al., 2018) are involved in ER stressmediated cell apoptosis. Conversely, PE_PGRS62 (Long et al., 2019) and PE_PGRS18 (Yang W. et al., 2017) can decrease apoptosis and enhance survival rate. PE31 increased guanylate-binding protein-1 (GBP-1) expression and inhibited caspase-3 activation and macrophage apoptosis through the NF- $\kappa \mathrm{B}$ pathway (Ali et al., 2020). Although apoptosis caused by some bacterial proteins favors bacterial survival, it also helps to kill intracellular bacteria and activate adaptive immunity (Schaible et al., 2003; Srinivasan et al., 2014). PE/PPE proteins with pro-apoptotic activity might serve as candidates for vaccine development.

Autophagy is related to autolysosome formation, which helps host cell clear out the pathogen, but an aberrant autolysosome may consume most cellular proteins and organelles, thus inducing autophagic cell death (Mariño et al., 2014). PE_ PGRS41 (Deng et al., 2017) and PE_PGRS47 (Saini et al., 2016) have been proved to inhibit autophagy from allowing pathogen survival. A recent report revealed that ubiquitinated PE_PGRS29 could recruit autophagy receptor p62 and deliver Mtb into autophagosomes. Disruption of the interaction between PE_PGRS29 and ubiquitin attenuates Mtb xenophagic clearance, leading to an enhanced bacterial load and an elevated inflammatory response (Chai et al., 2019).

Cell necrosis is involved in the dissemination and virulence of Mtb because it results in the release and spread of tuberculosiscausing pathogens (Behar et al., 2010). Such a function has been reported for PE25-PPE41 complex (Tundup et al., 2014), PE_PGRS33 (Dheenadhayalan et al., 2006), and PPE27 (Yang G. et al., 2017).
In addition, a PPE60-overexpressing strain has recently been found to increase intracebllular survival and shift cell fate to pyroptosis, a newly defined form of programmed cell death, which is correlated with restriction of intracellular growth and enhanced host immune response (Gong et al., 2019; Chai et al., 2020), and with the maturation of IL-1 $\beta$ and IL-18 (Beckwith et al., 2020).

\section{FUTURE APPLICATIONS OF PE/PPE FAMILY PROTEINS IN TB VACCINE DESIGN AND DIAGNOSTIC TOOL DEVELOPMENT}

Serological antibody assays are routinely performed; however, there is no gold standard in TB serological diagnosis. PE35, an RD1-encoded antigen, can significantly discriminate pulmonary or extra-pulmonary TB patients with healthy BCG-vaccinated individuals (Mukherjee et al., 2007). Another good example is PPE17, whose N-terminal induces high immunogenic response and had greater potential to be a sero-diagnostic marker than full-length PPE17 (Abraham et al., 2017), which could screen the latently infected subjects (Abraham et al., 2018). PPE2 may also serve as a serodiagnosis marker to detect the extra-pulmonary and smear-negative pulmonary cases (Abraham et al., 2014).

The highly immunogenic properties of $\mathrm{PE} / \mathrm{PPE}$ proteins have been demonstrated by the investigation of IFN $-\gamma \mathrm{T}$ cell responses generated during infection. $\mathrm{CD}^{+}{ }^{+}$-specific epitope-rich $\mathrm{PE} / \mathrm{PPE}$ proteins, including PE18, PE19, PPE25, PPE26, and PPE27, are potent inducers of cell-mediated immune responses (Sayes et al., 2012). Vordermeier et al. examined cellular immune responses against a panel of $36 \mathrm{PE} / \mathrm{PPE}$ proteins during human and bovine infection and observed that many were major targets of the cellular immune response to tuberculosis. The specific HLA$\mathrm{A}^{\star}$ 0201-restricted epitopes of PPE68 also elicit a potent cellular response (Duan et al., 2015). Additionally, the PE5 protein and EsxI have been proven as a diagnostic antigen of bovine tuberculosis during intradermal tests (Melo et al., 2015). A combination of PPE57 can also increase the sensitivity of ESAT- 6 or CFP-10 in the IFN- $\gamma$ releasing assay for detecting active TB (Chen et al., 2009). The highly cellular immune response indicates that $\mathrm{PE} / \mathrm{PPE}$ proteins may be better diagnostic and vaccine candidates (Vordermeier et al., 2012).

Numerous studies have also been carried out to assess the potential of PE/PPE proteins as candidate vaccine antigens. Several attempts seem promising. In dendritic cells, which serve as the most efficient antigen-presenting cells, PE27 (Kim et al., 2016), PPE39 (Choi et al., 2019), and PPE60 (Su et al., 2018) could change the cytokine profile toward a proinflammatory immune response, suggesting the possibility to be subunit vaccines for tuberculosis. In macrophages, PPE57 (Xu et al., 2015), PPE26 (Su et al., 2015), and PE3 (Singh et al., 2013) were also found to generate a protective immune response. Further, PPE44, HspX, and EsxV could enhance BCG protective efficacy (Mansury et al., 2019). Another vaccine candidate worth mentioning is the attenuated Mtbsppe25-pe19 
strain, which outcompeted BCG protective capacity (Sayes et al., 2012). Notably, the contribution of PE-specific and PPE-specific $\mathrm{T}$ helper cell 1 (Th1) effector cells in protective immunity against mycobacteria has been recently identified (Sayes et al., 2016).

However, there is a downside to the use of PE/PPE proteins in vaccines, as many of them are believed to hamper the host inflammatory response to evade immune surveillance, thus supporting the development of an immunopathological response. PE32/PPE65 (Khubaib et al., 2016), PPE37 (Daim et al., 2011), and PE25/PPE14 (Chen et al., 2015) were found to tilt the Th1 response toward a Th2 response, which favors the intracellular survival of bacteria. In addition, $\mathrm{PE} / \mathrm{PPE}$ proteins are polymorphic within clinical isolates (Hebert et al., 2007) and can be degradation-resistant, limiting MHC processing (Koh et al., 2009). However, researchers surprisingly found that the PPE18 protein, which upregulated IL-10 production (Nair et al., 2009) and inhibited the inflammatory response, could be explored as a therapeutic for sepsis caused by exaggerated inflammatory responses (Ahmed et al., 2018). Thorough characterization of candidates or exclusive use of the immunodominant epitopes of $\mathrm{PE} / \mathrm{PPE}$ proteins may facilitate vaccine development.

\section{DISCUSSION}

Since its discovery over 20 years ago, PE/PPE family has been recognized as exclusive to mycobacteria, especially in pathogenic species. Several studies have defined that PE/PPE protein expression is linked to ESX gene clusters is now well-established (Bottai et al., 2012; Sayes et al., 2012). Improved knowledge of the ESX system function has dramatically advanced our understanding of the biological function of specific $\mathrm{PE} / \mathrm{PPE}$ proteins. Moreover, structural biology studies have started to solve and explain the roles of protein complexes involved in PEPPE and ESX secretion (Ekiert and Cox, 2014). However, the biology and structure of $\mathrm{PE} / \mathrm{PPE}$ proteins remain far less understood than other mycobacterial proteins. Elucidating the structure of PE/PPE proteins and their complexes with ESX systems will be pivotal to a more comprehensive mechanistic understanding of how the PE/PPE protein family, in association with the ESX secretion system, contributes to the pathogenicity of Mtb. This is of importance for obtaining further insights into the virulence strategies of mycobacteria, and may provide novel targets for antimycobacterial treatment.

Another feature of the PE/PPE proteins is that they are often found as co-operonic pairs of mostly one PE- and one PPEcoding gene, whose products interact with each other (Akhter et al., 2012) and are believed to assemble as heterodimers (Strong

\section{REFERENCES}

Abraham, P. R., Latha, G. S., Valluri, V. L., and Mukhopadhyay, S. (2014). Mycobacterium tuberculosis PPE protein Rv0256c induces strong B cell response in tuberculosis patients. Infect. Genet. Evol. 22, 244-249. doi: 10.1016/j.meegid.2013.06.023

Abraham, P. R., Pathak, N., Pradhan, G., Sumanlatha, G., and Mukhopadhyay, S. (2017). The N-terminal domain of Mycobacterium tuberculosis PPE17 et al., 2006; Tundup et al., 2006; Tiwari et al., 2014). Such interactions have been predicted using bioinformatic tools (Riley et al., 2008) and proven through experimental evidence, as in the cases of PPE41 and PE25 (Tundup et al., 2006), PE35 and PPE68 (Tiwari et al., 2014), as well as PE19 and PPE51 (Wang et al., 2020). Korycka-Machała et al. found that PPE51 deletion rendered $\mathrm{Mtb}$ cells unable to replicate in propionamide, glucose, or glycerol. Further, some PE/PPE proteins, such as $\mathrm{PE} 20 / \mathrm{PPE} 31$ and PE32/PPE65, are required by Mtb during $\mathrm{Mg}^{2+}$ and $\mathrm{PO}^{2-}$ restriction (Wang et al., 2020). PPE36/PPE62 (Mitra et al., 2019) and PPE37 (Tullius et al., 2018) are essential for heme-iron acquisition and Mtb growth. Additionally, mutant PPE51 and PE19 strains developed resistance to 3bMP1, a compound with anti-tuberculosis activity (Wang et al., 2020). These data suggest that at least some PE/PPE proteins appear to act as solute-selective pores, allowing the access of exogenous agents or nutrients required for proliferation. Thus, focusing on genetic mutations of pe/ppe family members, which are often eliminated when analyzing next-generation sequencing data of clinically drug-resistant strains, may help discover antituberculosis drug resistance mechanisms. In summary, we believe that the PE/PPE family will remain a highly active area of research with various exciting features yet to be discovered.

\section{AUTHOR CONTRIBUTIONS}

JQ wrote the manuscript draft. XZ supervised and revised the manuscript. RC and HW gave some suggestions. All authors contributed to the article and approved the submitted version. Key scientific and technological projects of Xinjiang production and Construction Corps (2020AB015).

\section{FUNDING}

This work was supported by the National Natural Science Foundation of China (81673482 and 81971898), SKLGE-1912 and Key scientific and technological projects of Xinjiang production and Construction Corps (2020AB015).

\section{SUPPLEMENTARY MATERIAL}

The Supplementary Material for this article can be found online at: https://www.frontiersin.org/articles/10.3389/fcimb.2020. 594288/full\#supplementary-material. 
Animal Model of Sepsis. J. Immunol. 200, 3587-3598. doi: 10.4049/ jimmunol.1602065

Akhter, Y., Ehebauer, M. T., Mukhopadhyay, S., and Hasnain, S. E. (2012). The $\mathrm{PE} / \mathrm{PPE}$ multigene family codes for virulence factors and is a possible source of mycobacterial antigenic variation: Perhaps more? Biochimie 94, 110-116. doi: 10.1016/j.biochi.2011.09.026

Ali, M. K., Zhen, G., Nzungize, L., Stojkoska, A., Duan, X., Li, C., et al. (2020). Mycobacterium tuberculosis PE31 (Rv3477) Attenuates Host Cell Apoptosis and Promotes Recombinant M. smegmatis Intracellular Survival via Upregulating GTPase Guanylate Binding Protein-1. Front. Cell. Infect. Microbiol. 10:40. doi: 10.3389/fcimb.2020.00040

Bansal, K., Elluru, S. R., Narayana, Y., Chaturvedi, R., Patil, S. A., Kaveri, S. V., et al. (2010). PE_PGRS antigens of Mycobacterium tuberculosis induce maturation and activation of human dendritic cells. J. Immunol. Baltim. Md 1950 184, 3495-3504. doi: 10.4049/jimmunol.0903299

Basu, S., Pathak, S. K., Banerjee, A., Pathak, S., Bhattacharyya, A., Yang, Z., et al. (2007). Execution of Macrophage Apoptosis by PE_PGRS33 of Mycobacterium tuberculosis Is Mediated by Toll-like Receptor 2-dependent Release of Tumor Necrosis Factor- $\alpha$. J. Biol. Chem. 282, 1039-1050. doi: 10.1074/jbc.M604379200

Beckwith, K. S., Beckwith, M. S., Ullmann, S., Sætra, R. S., Kim, H., Marstad, A., et al. (2020). Plasma membrane damage causes NLRP3 activation and pyroptosis during Mycobacterium tuberculosis infection. Nat. Commun. 11, 2270. doi: 10.1038/s41467-020-16143-6

Behar, S. M., Divangahi, M., and Remold, H. G. (2010). Evasion of innate immunity by Mycobacterium tuberculosis: is death an exit strategy? Nat. Rev. Microbiol. 8, 668-674. doi: 10.1038/nrmicro2387

Bottai, D., Di Luca, M., Majlessi, L., Frigui, W., Simeone, R., Sayes, F., et al. (2012). Disruption of the ESX-5 system of Mycobacterium tuberculosis causes loss of PPE protein secretion, reduction of cell wall integrity and strong attenuation: ESX-5 of M. tuberculosis. Mol. Microbiol. 83, 1195-1209. doi: 10.1111/j.13652958.2012.08001.x

Brodin, P., Majlessi, L., Marsollier, L., de Jonge, M.II, Bottai, D., Demangel, C., et al. (2006). Dissection of ESAT-6 System 1 of Mycobacterium tuberculosis and Impact on Immunogenicity and Virulence. Infect. Immun. 74, 88-98. doi: 10.1128/IAI.74.1.88-98.2006

Chai, Q., Wang, X., Qiang, L., Zhang, Y., Ge, P., Lu, Z., et al. (2019). A Mycobacterium tuberculosis surface protein recruits ubiquitin to trigger host xenophagy. Nat. Commun. 10, 1973. doi: 10.1038/s41467-019-09955-8

Chai, Q., Wang, L., Liu, C. H., and Ge, B. (2020). New insights into the evasion of host innate immunity by Mycobacterium tuberculosis. Cell. Mol. Immunol. 17, 901-913. doi: 10.1038/s41423-020-0502-z

Chen, J., Su, X., Zhang, Y., Wang, S., Shao, L., Wu, J., et al. (2009). Novel recombinant RD2- and RD11-encoded Mycobacterium tuberculosis antigens are potential candidates for diagnosis of tuberculosis infections in BCGvaccinated individuals. Microbes Infect. 11, 876-885. doi: 10.1016/ j.micinf.2009.05.008

Chen, J. M., Zhang, M., Rybniker, J., Boy-Röttger, S., Dhar, N., Pojer, F., et al. (2013). Mycobacterium tuberculosis EspB binds phospholipids and mediates EsxA-independent virulence: EsxA-independent role of EspB in $M . t b$ virulence. Mol. Microbiol. 89, 1154-1166. doi: 10.1111/mmi.12336

Chen, W., Bao, Y., Chen, X., Burton, J., Gong, X., Gu, D., et al. (2015). Mycobacterium tuberculosis PE25/PPE41 protein complex induces activation and maturation of dendritic cells and drives Th2-biased immune responses. Med. Microbiol. Immunol. (Berl.) 205, 119-131. doi: 10.1007/ s00430-015-0434-x

Choi, H.-H., Kwon, K. W., Han, S. J., Kang, S. M., Choi, E., Kim, A., et al. (2019). PPE39 of the Mycobacterium tuberculosis strain Beijing/K induces Th1-cell polarization through dendritic cell maturation. J. Cell Sci. 132, jcs228700. doi: $10.1242 /$ jcs. 228700

Daim, S., Kawamura, I., Tsuchiya, K., Hara, H., Kurenuma, T., Shen, Y., et al. (2011). Expression of the Mycobacterium tuberculosis PPE37 protein in Mycobacterium smegmatis induces low tumour necrosis factor alpha and interleukin 6 production in murine macrophages. J. Med. Microbiol. 60, 582591. doi: 10.1099/jmm.0.026047-0

Daleke, M. H., Ummels, R., Bawono, P., Heringa, J., Vandenbroucke-Grauls, C. M. J. E., Luirink, J., et al. (2012). General secretion signal for the mycobacterial type VII secretion pathway. Proc. Natl. Acad. Sci. 109, 11342-11347. doi: $10.1073 /$ pnas. 1119453109
Deng, W., Li, W., Zeng, J., Zhao, Q., Li, C., Zhao, Y., et al. (2014). Mycobacterium Tuberculosis PPE Family Protein Rv1808 Manipulates Cytokines Profile via

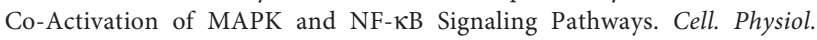
Biochem. 33, 273-288. doi: 10.1159/000356668

Deng, W., Yang, W., Zeng, J., Abdalla, A. E., and Xie, J. (2016). Mycobacterium tuberculosis PPE32 promotes cytokines production and host cell apoptosis through caspase cascade accompanying with enhanced ER stress response. Oncotarget 7, 67347-67359. doi: 10.18632/oncotarget.12030

Deng, W., Long, Q., Zeng, J., Li, P., Yang, W., Chen, X., et al. (2017). Mycobacterium tuberculosis PE_PGRS41 Enhances the Intracellular Survival of M. smegmatis within Macrophages Via Blocking Innate Immunity and Inhibition of Host Defense. Sci. Rep. 7:46716. doi: 10.1038/srep46716

Dheenadhayalan, V., Delogu, G., and Brennan, M. J. (2006). Expression of the PE_PGRS 33 protein in Mycobacterium smegmatis triggers necrosis in macrophages and enhanced mycobacterial survival. Microbes Infect. 8, 262272. doi: 10.1016/j.micinf.2005.06.021

Dong, D., Wang, D., Li, M., Wang, H., Yu, J., Wang, C., et al. (2012). PPE38 Modulates the Innate Immune Response and Is Required for Mycobacterium marinum Virulence. Infect. Immun. 80, 43-54. doi: 10.1128/IAI.05249-11

Duan, L., Gan, H., Golan, D. E., and Remold, H. G. (2002). Critical Role of Mitochondrial Damage in Determining Outcome of Macrophage Infection with Mycobacterium tuberculosis. J. Immunol. 169, 5181-5187. doi: 10.4049/ jimmunol.169.9.5181

Duan, Z., Li, Q., Wang, S., Chen, X., Liu, H., Chen, B., et al. (2015). Identification of Mycobacterium tuberculosis PPE68-Specific HLA-A ${ }^{*} 0201$-Restricted Epitopes for Tuberculosis Diagnosis. Curr. Microbiol. 70, 769-778. doi: $10.1007 / \mathrm{s} 00284-015-0786-\mathrm{x}$

Ekiert, D. C., and Cox, J. S. (2014). Structure of a PE-PPE-EspG complex from Mycobacterium tuberculosis reveals molecular specificity of ESX protein secretion. Proc. Natl. Acad. Sci. 111, 14758-14763. doi: 10.1073/pnas.1409345111

Fink, S. L., and Cookson, B. T. (2005). Apoptosis, Pyroptosis, and Necrosis: Mechanistic Description of Dead and Dying Eukaryotic Cells. Infect. Immun. 73, 1907-1916. doi: 10.1128/IAI.73.4.1907-1916.2005

Fishbein, S., van Wyk, N., Warren, R. M., and Sampson, S. L. (2015). Phylogeny to function: PE/PPE protein evolution and impact on $M$ ycobacterium tuberculosis pathogenicity: Evolution of PE/PPE-associated virulence. Mol. Microbiol. 96, 901-916. doi: 10.1111/mmi.12981

Gey van Pittius, N. C., Sampson, S. L., Lee, H., Kim, Y., van Helden, P. D., and Warren, R. M. (2006). Evolution and expansion of the Mycobacterium tuberculosis PE and PPE multigene families and their association with the duplication of the ESAT-6 (esx) gene cluster regions. BMC Evol. Biol. 6:95. doi: 10.1186/1471-2148-6-95

Gong, Z., Kuang, Z., Li, H., Li, C., Ali, M. K., Huang, F., et al. (2019). Regulation of host cell pyroptosis and cytokines production by Mycobacterium tuberculosis effector PPE60 requires LUBAC mediated NF-KB signaling. Cell. Immunol. 335, 41-50. doi: 10.1016/j.cellimm.2018.10.009

Grover, S., Sharma, T., Singh, Y., Kohli, S., P, M., Singh, A., et al. (2018). The PGRS Domain of Mycobacterium tuberculosis PE_PGRS Protein Rv0297 Is Involved in Endoplasmic Reticulum Stress-Mediated Apoptosis through Toll-Like Receptor 4. mBio 9, e01017-e01018. doi: 10.1128/mBio.01017-18

Hebert, A. M., Talarico, S., Yang, D., Durmaz, R., Marrs, C. F., Zhang, L., et al. (2007). DNA Polymorphisms in the pepA and PPE18 Genes among Clinical Strains of Mycobacterium tuberculosis: Implications for Vaccine Efficacy. Infect. Immun. 75, 5798-5805. doi: 10.1128/IAI.00335-07

Huang, Y., Zhou, X., Bai, Y., Yang, L., Yin, X., Wang, Z., et al. (2012). Phagolysosome maturation of macrophages was reduced by PE_PGRS 62 protein expressing in Mycobacterium smegmatis and induced in IFN- $\gamma$ priming. Vet. Microbiol. 160, 117-125. doi: 10.1016/j.vetmic.2012.05.011

Iantomasi, R., Sali, M., Cascioferro, A., Palucci, I., Zumbo, A., Soldini, S., et al. (2012). PE_PGRS30 is required for the full virulence of Mycobacterium tuberculosis: PE_PGRS30 is an Mtb virulence factor. Cell. Microbiol. 14, 356-367. doi: 10.1111/j.1462-5822.2011.01721.x

Jha, S. S., Danelishvili, L., Wagner, D., Maser, J., Li, Y., Moric, I., et al. (2010). Virulence-related Mycobacterium avium subsp hominissuis MAV_2928 gene is associated with vacuole remodeling in macrophages. BMC Microbiol. 10:100. doi: 10.1186/1471-2180-10-100

Jiang, Y., Wei, J., Liu, H., Li, G., Guo, Q., Qiu, Y., et al. (2016). Polymorphisms in the PE35 and PPE68 antigens in Mycobacterium tuberculosis strains may affect 
strain virulence and reflect ongoing immune evasion. Mol. Med. Rep. 13, 947954. doi: $10.3892 / \mathrm{mmr} .2015 .4589$

Khubaib, M., Sheikh, J. A., Pandey, S., Srikanth, B., Bhuwan, M., Khan, N., et al. (2016). Mycobacterium tuberculosis Co-operonic PE32/PPE65 Proteins Alter Host Immune Responses by Hampering Th1 Response. Front. Microbiol. 7, 719. doi: $10.3389 /$ fmicb. 2016.00719

Kim, W. S., Kim, J.-S., Cha, S. B., Kim, S. J., Kim, H., Kwon, K. W., et al. (2016). Mycobacterium tuberculosis PE27 activates dendritic cells and contributes to Th1-polarized memory immune responses during in vivo infection. Immunobiology 221, 440-453. doi: 10.1016/j.imbio.2015.11.006

Koh, K. W., Lehming, N., and Seah, G. T. (2009). Degradation-resistant protein domains limit host cell processing and immune detection of mycobacteria. Mol. Immunol. 46, 1312-1318. doi: 10.1016/j.molimm.2008.11.008

Korotkova, N., Freire, D., Phan, T. H., Ummels, R., Creekmore, C. C., Evans, T. J., et al. (2014). Structure of the $M$ ycobacterium tuberculosis type VII secretion system chaperone EspG ${ }_{5}$ in complex with PE25-PPE41 dimer: Type VII secretion chaperone and PE-PPE dimer. Mol. Microbiol. 94, 367-382. doi: $10.1111 / \mathrm{mmi} .12770$

Li, H., Li, Q., Yu, Z., Zhou, M., and Xie, J. (2016). Mycobacterium tuberculosis PE13 (Rv1195) manipulates the host cell fate via p38-ERK-NF- $\kappa B$ axis and apoptosis. Apoptosis 21, 795-808. doi: 10.1007/s10495-016-1249-y

Li, W., Deng, W., and Xie, J. (2019). Expression and regulatory networks of Mycobacterium tuberculosis PE/PPE family antigens. J. Cell. Physiol. 234, 77427751. doi: $10.1002 /$ jcp. 27608

Long, Q., Xiang, X., Yin, Q., Li, S., Yang, W., Sun, H., et al. (2019). PE_PGRS62 promotes the survival of Mycobacterium smegmatis within macrophages via disrupting ER stress-mediated apoptosis. J. Cell. Physiol. 234, 19774-19784. doi: $10.1002 /$ jcp. 28577

Majlessi, L., Prados-Rosales, R., Casadevall, A., and Brosch, R. (2015). Release of mycobacterial antigens. Immunol. Rev. 264, 25-45. doi: 10.1111/imr.12251

Mansury, D., Ghazvini, K., Amel Jamehdar, S., Badiee, A., Tafaghodi, M., Nikpoor, A. R., et al. (2019). Enhancement of the effect of BCG vaccine against tuberculosis using DDA/TDB liposomes containing a fusion protein of HspX, PPE44, and EsxV. Artif. Cells Nanomed Biotechnol. 47, 370-377. doi: 10.1080/21691401.2018.1557674

Mariño, G., Niso-Santano, M., Baehrecke, E. H., and Kroemer, G. (2014). Selfconsumption: the interplay of autophagy and apoptosis. Nat. Rev. Mol. Cell Biol. 15, 81-94. doi: 10.1038/nrm3735

McGuire, A., Weiner, B., Park, S., Wapinski, I., Raman, S., Dolganov, G., et al. (2012). Comparative analysis of mycobacterium and related actinomycetes yields insight into the evolution of mycobacterium tuberculosis pathogenesis. BMC Genomics 13:120. doi: 10.1186/1471-2164-13-120

Meena, L. S., and Meena, J. (2016). Cloning and characterization of a novel PE_PGRS60 protein (Rv3652) of Mycobacterium tuberculosis $\mathrm{H}_{37} \mathrm{Rv}$ exhibit fibronectin-binding property: New FnBP from M. tuberculosis. Biotechnol. Appl. Biochem. 63, 525-531. doi: 10.1002/bab.1411

Melo, E., Souza, I., Ramos, C., Osório, A., Verbisck, N., and Araújo, F. (2015). Evaluation of the use of recombinant proteins of Mycobacterium bovis as antigens in intradermal tests for diagnosis of bovine tuberculosis. Arch. Med. Vet. 47, 273-280. doi: 10.4067/S0301-732X2015000300003

Meng, L., Tong, J., Wang, H., Tao, C., Wang, Q., Niu, C., et al. (2017). PPE38 Protein of Mycobacterium tuberculosis Inhibits Macrophage MHC Class I Expression and Dampens CD8+ T Cell Responses. Front. Cell. Infect. Microbiol. 7, 68. doi: 10.3389/fcimb.2017.00068

Mitra, A., Ko, Y.-H., Cingolani, G., and Niederweis, M. (2019). Heme and hemoglobin utilization by Mycobacterium tuberculosis. Nat. Commun. 10, 4260. doi: 10.1038/s41467-019-12109-5

Mukherjee, P., Dutta, M., Datta, P., Dasgupta, A., Pradhan, R., Pradhan, M., et al. (2007). The RD1-encoded antigen Rv3872 of Mycobacterium tuberculosis as a potential candidate for serodiagnosis of tuberculosis. Clin. Microbiol. Infect. 13, 146-152. doi: 10.1111/j.1469-0691.2006.01660.x

Nair, S., Ramaswamy, P. A., Ghosh, S., Joshi, D. C., Pathak, N., Siddiqui, I., et al. (2009). The PPE18 of Mycobacterium tuberculosis Interacts with TLR2 and Activates IL-10 Induction in Macrophage. J. Immunol. 183, 6269-6281. doi: 10.4049/jimmunol.0901367

Nair, S., Pandey, A. D., and Mukhopadhyay, S. (2011). The PPE18 Protein of Mycobacterium tuberculosis Inhibits NF- $\mathrm{kB} /$ rel-Mediated Proinflammatory Cytokine Production by Upregulating and Phosphorylating Suppressor of
Cytokine Signaling 3 Protein. J. Immunol. 186, 5413-5424. doi: 10.4049/ jimmunol.1000773

Palucci, I., Camassa, S., Cascioferro, A., Sali, M., Anoosheh, S., Zumbo, A., et al. (2016). PE_PGRS33 Contributes to Mycobacterium tuberculosis Entry in Macrophages through Interaction with TLR2. PLoS One 11, e0150800. doi: 10.1371/journal.pone. 0150800

Peng, X., Luo, T., Zhai, X., Zhang, C., Suo, J., Ma, P., et al. (2018). PPE11 of Mycobacterium tuberculosis can alter host inflammatory response and trigger cell death. Microb. Pathog. 126, 45-55. doi: 10.1016/j.micpath.2018.10.031

Qureshi, R., Rameshwaram, N. R., Battu, M. B., and Mukhopadhyay, S. (2019). PPE65 of M. tuberculosis regulate pro-inflammatory signalling through LRR domains of Toll like receptor-2. Biochem. Biophys. Res. Commun. 508, 152158. doi: 10.1016/j.bbrc.2018.11.094

Ramakrishnan, P., Aagesen, A. M., McKinney, J. D., and Tischler, A. D. (2016). Mycobacterium tuberculosis Resists Stress by Regulating PE19 Expression. Infect. Immun. 84, 735-746. doi: 10.1128/IAI.00942-15

Rastogi, S., Singh, A. K., Pant, G., Mitra, K., Sashidhara, K. V., and Krishnan, M. Y. (2017). Down-regulation of PE11, a cell wall associated esterase, enhances the biofilm growth of Mycobacterium tuberculosis and reduces cell wall virulence lipid levels. Microbiology 163, 52-61. doi: 10.1099/mic.0.000417

Riley, R., Pellegrini, M., and Eisenberg, D. (2008). Identifying Cognate Binding Pairs among a Large Set of Paralogs: The Case of PE/PPE Proteins of Mycobacterium tuberculosis. PLoS Comput. Biol. 4, e1000174. doi: 10.1371/ journal.pcbi.1000174

Saini, N. K., Baena, A., Ng, T. W., Venkataswamy, M. M., Kennedy, S. C., Kunnath-Velayudhan, S., et al. (2016). Suppression of autophagy and antigen presentation by Mycobacterium tuberculosis PE_PGRS47. Nat. Microbiol. 1, 16133. doi: 10.1038/nmicrobiol.2016.133

Sassetti, C. M., and Rubin, E. J. (2003). Genetic requirements for mycobacterial survival during infection. Proc. Natl. Acad. Sci. 100, 12989-12994. doi: 10.1073/ pnas. 2134250100

Sayes, F., Sun, L., Di Luca, M., Simeone, R., Degaiffier, N., Fiette, L., et al. (2012). Strong Immunogenicity and Cross-Reactivity of Mycobacterium tuberculosis ESX-5 Type VII Secretion -Encoded PE-PPE Proteins Predicts Vaccine Potential. Cell Host Microbe 11, 352-363. doi: 10.1016/j.chom.2012.03.003

Sayes, F., Pawlik, A., Frigui, W., Gröschel, M.II, Crommelynck, S., Fayolle, C., et al. (2016). CD4+ T Cells Recognizing PE/PPE Antigens Directly or via Cross Reactivity Are Protective against Pulmonary Mycobacterium tuberculosis Infection. PLoS Pathog. 12, e1005770. doi: 10.1371/journal.ppat.1005770

Schaible, U. E., Winau, F., Sieling, P. A., Fischer, K., Collins, H. L., Hagens, K., et al. (2003). Apoptosis facilitates antigen presentation to T lymphocytes through MHC-I and CD1 in tuberculosis. Nat. Med. 9, 1039-1046. doi: 10.1038/nm906 Serafini, A., Boldrin, F., Palu, G., and Manganelli, R. (2009). Characterization of a Mycobacterium tuberculosis ESX-3 Conditional Mutant: Essentiality and Rescue by Iron and Zinc. J. Bacteriol. 191, 6340-6344. doi: 10.1128/ JB.00756-09

Siegrist, M. S., Unnikrishnan, M., McConnell, M. J., Borowsky, M., Cheng, T.-Y., Siddiqi, N., et al. (2009). Mycobacterial Esx-3 is required for mycobactinmediated iron acquisition. Proc. Natl. Acad. Sci. 106, 18792-18797. doi: 10.1073/pnas.0900589106

Singh, S. K., Kumari, R., Singh, D. K., Tiwari, S., Singh, P. K., Sharma, S., et al. (2013). Putative roles of a proline-glutamic acid-rich protein (PE3) in intracellular survival and as a candidate for subunit vaccine against Mycobacterium tuberculosis. Med. Microbiol. Immunol. (Berl.) 202, 365-377. doi: 10.1007/s00430-013-0299-9

Srinivasan, L., Ahlbrand, S., and Briken, V. (2014). Interaction of Mycobacterium tuberculosis with Host Cell Death Pathways. Cold Spring Harb. Perspect. Med. 4, a022459-a022459. doi: 10.1101/cshperspect.a022459

Strong, M., Sawaya, M. R., Wang, S., Phillips, M., Cascio, D., and Eisenberg, D. (2006). Toward the structural genomics of complexes: Crystal structure of a PE/PPE protein complex from Mycobacterium tuberculosis. Proc. Natl. Acad. Sci. 103, 8060-8065. doi: 10.1073/pnas.0602606103

Su, H., Kong, C., Zhu, L., Huang, Q., Luo, L., Wang, H., et al. (2015). PPE26 induces TLR2-dependent activation of macrophages and drives Th1-type Tcell immunity by triggering the cross-talk of multiple pathways involved in the host response. Oncotarget 6, 38517-38537. doi: 10.18632/oncotarget.5956

Su, H., Zhang, Z., Liu, Z., Peng, B., Kong, C., Wang, H., et al. (2018). Mycobacterium tuberculosis PPE60 antigen drives Th1/Th17 responses via 
Toll-like receptor 2-dependent maturation of dendritic cells. J. Biol. Chem. 293, 10287-10302. doi: 10.1074/jbc.RA118.001696

Thi, E. P., Hong, C. J. H., Sanghera, G., and Reiner, N. E. (2013). Identification of the Mycobacterium tuberculosis protein PE-PGRS62 as a novel effector that functions to block phagosome maturation and inhibit iNOS expression: PEPGRS62 modulates macrophage function. Cell. Microbiol. 15, 795-808. doi: $10.1111 / \mathrm{cmi} .12073$

Tiwari, B., Soory, A., and Raghunand, T. R. (2014). An immunomodulatory role for the Mycobacterium tuberculosis region of difference 1 locus proteins PE35 (Rv3872) and PPE68 (Rv3873). FEBS J. 281, 1556-1570. doi: 10.1111/febs.12723

Tiwari, B., Ramakrishnan, U. M., and Raghunand, T. R. (2015). The $M$ ycobacterium tuberculosis protein pair PE9 (Rv1088)-PE10 (Rv1089) forms heterodimers and induces macrophage apoptosis through Toll-like receptor 4: The PE9-PE10 protein pair of M. tb is a TLR4 ligand. Cell. Microbiol. 17, 16531669. doi: $10.1111 / \mathrm{cmi} .12462$

Tufariello, J. M., Chapman, J. R., Kerantzas, C. A., Wong, K.-W., Vilchèze, C., Jones, C. M., et al. (2016). Separable roles for Mycobacterium tuberculosis ESX3 effectors in iron acquisition and virulence. Proc. Natl. Acad. Sci. 113, E348E357. doi: 10.1073/pnas.1523321113

Tullius, M. V., Nava, S., and Horwitz, M. A. (2018). PPE37 Is Essential for Mycobacterium tuberculosis Heme-Iron Acquisition (HIA), and a Defective PPE37 in Mycobacterium bovis BCG Prevents HIA. Infect. Immun. 87, e00540e00518. doi: 10.1128/IAI.00540-18. /iai/87/2/IAI.00540-18.atom.

Tundup, S., Akhter, Y., Thiagarajan, D., and Hasnain, S. E. (2006). Clusters of PE and PPE genes of Mycobacterium tuberculosis are organized in operons: Evidence that PE Rv2431c is co-transcribed with PPE Rv2430c and their gene products interact with each other. FEBS Lett. 580, 1285-1293. doi: 10.1016/j.febslet.2006.01.042

Tundup, S., Mohareer, K., and Hasnain, S. E. (2014). Mycobacterium tuberculosis PE25/PPE41 protein complex induces necrosis in macrophages: Role in virulence and disease reactivation? FEBS Open Bio 4, 822-828. doi: 10.1016/ j.fob.2014.09.001

Vordermeier, H. M., Hewinson, R. G., Wilkinson, R. J., Wilkinson, K. A., Gideon, H. P., Young, D. B., et al. (2012). Conserved Immune Recognition Hierarchy of Mycobacterial PE/PPE Proteins during Infection in Natural Hosts. PLoS One 7, e40890. doi: 10.1371/journal.pone.0040890

Wang, Q., Boshoff, H.II, Harrison, J. R., Ray, P. C., Green, S. R., Wyatt, P. G., et al. (2020). PE/PPE proteins mediate nutrient transport across the outer membrane of Mycobacterium tuberculosis. Science 367, 1147-1151. doi: $10.1126 /$ science.aav5912

Xu, Y., Yang, E., Huang, Q., Ni, W., Kong, C., Liu, G., et al. (2015). PPE57 induces activation of macrophages and drives Th1-type immune responses through TLR2. J. Mol. Med. 93, 645-662. doi: 10.1007/s00109-014-1243-1

Yang, G., Luo, T., Sun, C., Yuan, J., Peng, X., Zhang, C., et al. (2017). PPE27 in Mycobacterium smegmatis Enhances Mycobacterial Survival and Manipulates Cytokine Secretion in Mouse Macrophages. J. Interferon Cytokine Res. 37, 421431. doi: $10.1089 /$ jir.2016.0126

Yang, W., Deng, W., Zeng, J., Ren, S., Ali, M. K., Gu, Y., et al. (2017). Mycobacterium tuberculosis PE_PGRS18 enhances the intracellular survival of M. smegmatis via altering host macrophage cytokine profiling and attenuating the cell apoptosis. Apoptosis 22, 502-509. doi: 10.1007/s10495016-1336-0

Yu, Z., Zhang, C., Zhou, M., Li, Q., Li, H., Duan, W., et al. (2017). Mycobacterium tuberculosis PPE44 (Rv2770c) is involved in response to multiple stresses and promotes the macrophage expression of IL-12 p40 and IL-6 via the p38, ERK, and NF-KB signaling axis. Int. Immunopharmacol. 50, 319-329. doi: 10.1016/ j.intimp.2017.06.028

Yu, X., Feng, J., Huang, L., Gao, H., Liu, J., Bai, S., et al. (2019). Molecular Basis Underlying Host Immunity Subversion by Mycobacterium tuberculosis PE/ PPE Family Molecules. DNA Cell Biol. 38, 1178-1187. doi: 10.1089/ dna.2019.4852

Zumbo, A., Palucci, I., Cascioferro, A., Sali, M., Ventura, M., D’Alfonso, P., et al. (2013). Functional dissection of protein domains involved in the immunomodulatory properties of PE_PGRS33 of Mycobacterium tuberculosis. Pathog. Dis. 69, 232-239. doi: 10.1111/2049-632X.12096

Conflict of Interest: The authors declare that the research was conducted in the absence of any commercial or financial relationships that could be construed as a potential conflict of interest.

Copyright ( 2020 Qian, Chen, Wang and Zhang. This is an open-access article distributed under the terms of the Creative Commons Attribution License (CC BY). The use, distribution or reproduction in other forums is permitted, provided the original author(s) and the copyright owner(s) are credited and that the original publication in this journal is cited, in accordance with accepted academic practice. No use, distribution or reproduction is permitted which does not comply with these terms. 